\title{
THE STARBURST PHENOMENON FROM THE OPTICAL/NEAR-IR PERSPECTIVE
}

\author{
Nils Bergvall, ${ }^{1}$ Thomas Marquart, ${ }^{1}$ Göran Östlin, ${ }^{2}$ Erik Zackrisson ${ }^{1}$ \\ ${ }^{1}$ Uppsala Astronomical Observatory \\ Box 515, SE-751 20 Uppsala, Sweden \\ nils.bergvall@astro.uu.se, thomas.marquart@astro.uu.se, ez@astro.uu.se \\ ${ }^{2}$ Stockholm Observatory \\ AlbaNova University Center, Roslagstullsbacken 21, SE-106 91 Stockholm, Sweden \\ ostlin@astro.su.se
}

\begin{abstract}
The optical/near-IR stellar continuum carries unique information about the stellar population in a galaxy, its mass function and star-formation history. Star-forming regions display rich emission-line spectra from which we can derive the dust and gas distribution, map velocity fields, metallicities and young massive stars and locate shocks and stellar winds. All this information is very useful in the dissection of the starburst phenomenon. We discuss a few of the advantages and limitations of observations in the optical/near-IR region and focus on some results. Special attention is given to the role of interactions and mergers and observations of the relatively dust-free starburst dwarfs. In the future we expect new and refined diagnostic tools to provide us with more detailed information about the IMF, strength and duration of the burst and its triggering mechanisms.
\end{abstract}

Keywords: galaxies:dwarfs, galaxies:evolution, galaxies:interactions, galaxies:starburst, infrared:galaxies

\section{Introduction}

Optical/near-IR broadband photometry of a starburst galaxy gives a first indication of burst strength, age and distribution of the young and old populations and their basic morphological structure parameters. Model based spectrophotometric tools are provided for more detailed analysis. A rich set of emission lines are used for analysis of kinematics, chemical abundance, shocks, stellar upper mass limit and distribution of dust and molecular gas. Absorption line indices provide estimates of age 
and IMF of the evolved population. Fig. 1 shows a synthetic spectrum a mixture of a young and old population with a mass ratio 2:1. A general review of the diagnostic tools and the limitations of the photoionization models used in the analysis is discussed by Schaerer (2001).

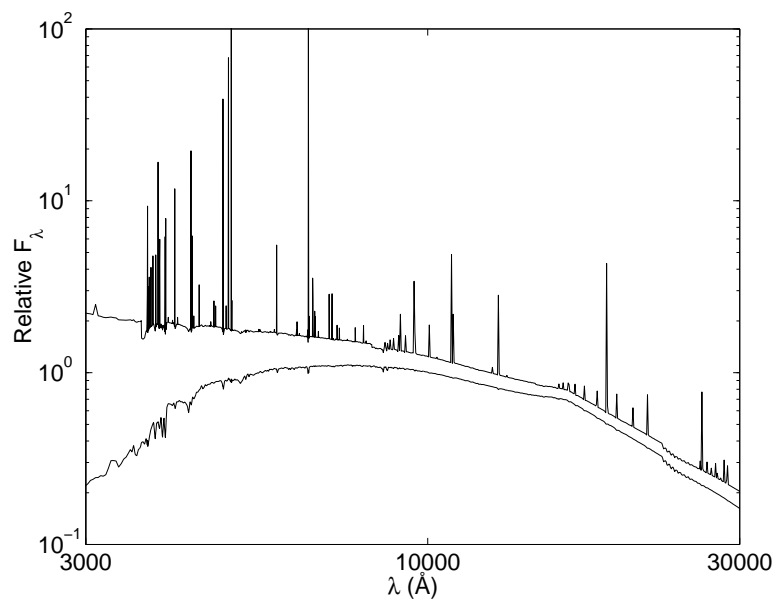

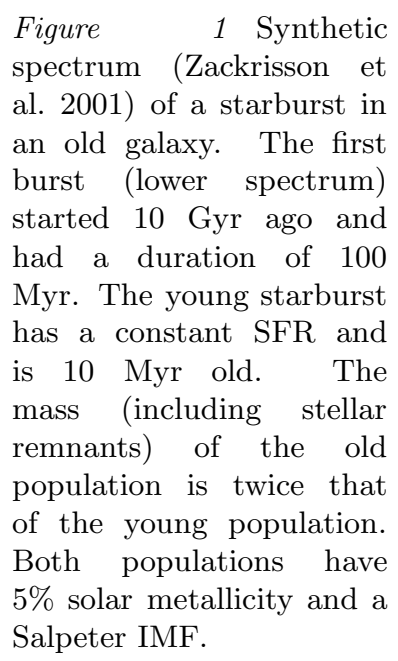
spectrum (Zackrisson et al. 2001) of a starburst in an old galaxy. The first burst (lower spectrum) started 10 Gyr ago and had a duration of 100 Myr. The young starburst has a constant SFR and is 10 Myr old. The mass (including stellar remnants) of the old population is twice that of the young population. Both populations have $5 \%$ solar metallicity and a Salpeter IMF.

Heavy dust obscuration, in particular in LIRGs and ULIRGs, has been a problem in the optical/near-IR. Here we will therefore focus on starbursts in low-extinction regions, notably starburst dwarf galaxies. First, however, we will discuss a widely debated issue where optical data originally had a strong impact, namely the importance of gravitational interactions as a starburst triggering mechanism.

\section{Starbursts and tidal interaction}

It is clear from the properties of ULIRGs that mergers are required to trigger major starbursts. But is it a sufficient requirement? How often do mergers and close encounters generate starbursts? To answer this question it is common to compare two galaxy samples - interacting/merging galaxies (IGs) or pairs, and non-interacting galaxies (NIGs). A problem with the comparison is that NIGs and IGs have evolved in different environments where e.g. mergers, ram pressure, harassment and gas infall have different influence. Integrated broadband photometry and $\mathrm{H} \alpha$ emission are the most widely used tools in this context. In the classical paper by Larson \& Tinsley (1978) the authors claim, based on UBV data, that interactions frequently trigger a major SF increase involving as much as $5 \%$ of the total mass. Many follow-up studies seem to confirm the result but are often influenced by strong selection effects, non-matching morphological type distribution NIGs/IGs and are focus- 
ing on the most dramatic cases. Studies based on more well constrained samples (Bergvall et al. 2002, Brosch et al. 2004) do not confirm these results but find that tidal interactions have an insignificant influence on the SF history of galaxies in the local universe. There seems to be an agreement however, of a correlation between interaction and increased SF within the central kpc (first discussed by Keel et al. 1985). Galaxy pairs with small separations show similar trends as seen in $\mathrm{H} \alpha$ (Barton et al. 2000, Lambas et al. 2003, Nikolic et al. 2004). The mean increase is in both cases is quite moderate however, and few cases are qualified to be called 'nuclear starbursts'. Bergvall et al. (2000) and Varela et al. (2004) find that masses of perturbed galaxies are higher than NIGs of similar morphology indicating that they experience mergers more frequently. This may lead to a steady inflow of gas that can explain part of the increased SF in the centre. Varela et al. also find a higher frequency of bars in disturbed systems, in accordance with related studies in the past (see Knapen 2004). Bars are known to generate mass inflows. Thus it is not clear what is the main triggering mechanism of the central increase in SF. The conclusion must be that there is no strong support that tidal interactions generate starburst activity that significantly affects the SF history of galaxies in the local universe. Estimates give room for major starbursts among less than a few \% of the IGs.

\section{Blue compact galaxies}

Blue compact galaxies (BCGs) is a not well defined type as the galaxies are selected either from spectroscopic or photometric critera. The general properties are high surface brightness, low chemical abundance and a high gas mass fraction. They have a wide range of morphologies (Loose \& Thuan 1986). Are they bursting? Fig. 2 shows $\mathrm{L}_{B} / \mathcal{M}_{\mathrm{HI}}$ vs. $\mathrm{M}_{B}$ of different types of gas rich galaxies. The BCG sample is incomplete but constitutes a representative part of the nearby sample of starburst dwarfs (Mrk, UM, Tololo etc.). We see that there is a continuous distribution towards high $\mathrm{L}_{B} / \mathcal{M}_{\mathrm{HI}}$ but that the properties of most BCGs are similar to dIrr and late type spirals of similar luminosity, i.e. they are probably not bursting. The high surface brightness of the burst could be due to a high column density (and a small scalelength, cf. Papaderos et al. 1996 and Salzer et al. 2002), perhaps caused by a low angular momentum. Since their gas mass often constitutes a major fraction of the total mass (Salzer et al. 2002), the diagram shows that starbursts in these galaxies are either shortlived or rare.

Some BCGs have a $\sim$ tenfold global increase in SFR, i.e they are true starbursts. What are their specific properties? There is no strong 


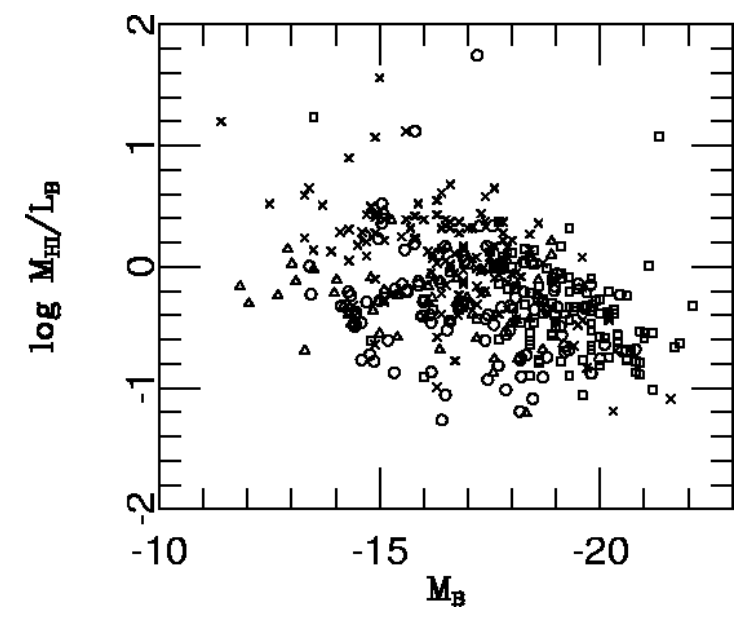

Figure 2 The hydrogen mass per B luminosity as function of absolute B magnitude for four different types of galaxies: BCGs (circles), dIrr (triangles), LSB galaxies (crosses) and late type spiral galaxies (squares). The data are obtained from different sources in the literature. It is incomplete but estimated to be representative.

indication of a correlation between SF activity and tidal interactions (Brosch 2004, Hunter and Elmegreen 2004). On the other hand BCGs appear to be involved in mergers with intense SF more frequently than other dwarfish galaxies (e.g. Gil de Paz 2004). It could indicate that mergers are important triggers and morphologically shortlived. The gas consumption rates are typically shorter than $100 \mathrm{Myr}$, i.e. similar to the dynamical timescale of a merger.

Ages and masses. Dynamical mass estimates of BCGs are difficult since the kinematics sometimes are quite chaotic due to the mass motions that cause the burst and because of the SN winds. To overcome the problem with the stellar winds it becomes necessary to use stellar absorption features. The only useful lines for this purpose are the Ca II triplet lines at about $8500 \AA$. Not until quite recently has this option become accessible (Östlin et al. 2004). The results are very promising and will soon help to solve the question regarding the coupling between gas and stars and facilitate the detailed analysis of velocity fields based on $\mathrm{H} \alpha$ (e.g. Marquart et al. 2004).

Age and SFR are often estimated from the $\mathrm{H} \alpha$ flux, the $\mathrm{H} \alpha$ equivalent width $(\mathrm{EW}(\mathrm{H} \alpha))$ and broadband photometry. From this the 'photometric mass' is obtained assuming that the SFR is constant. The age is however difficult to determine, even if we assume that the SFR is constant. In such a case, $\mathrm{EW}(\mathrm{H} \alpha)$ is a function of the IMF and age. The IMF slope in starbursts seems to be well constrained in the intermediate stellar mass range (Elmegreen 2004) but not so well for high masses. Fig 3 shows the predicted $\mathrm{EW}(\mathrm{H} \alpha)$ for two values of the upper mass limit, 40 and 120 solar masses. It can be seen that the predicted ages differ with 
a factor of 5-10 over a large age range. There is also an observational problem in that intense starbursts may have huge Strömgrenspheres from which the $\mathrm{H} \alpha$ emission may be lost due to a limited aperture size. The uncertainty in the determination of the widely used $b$ parameter $(b=$ $\mathrm{SFR} /<\mathrm{SFR}>$ ) obviously must be quite high, in particular if we consider the poorly constrained SF history.

For BCGs there seems to be a simple way to account for the SF history reasonably well. It is based on a two component model of the galaxy consisting of a starburst superposed on a host galaxy with an exponential luminosity profile. If photometric masses are applied to this model we find that there a fairly tight correlation between mass and central velocity dispersion (Östlin et al. 2001), indicating that this simple model is quite successful.

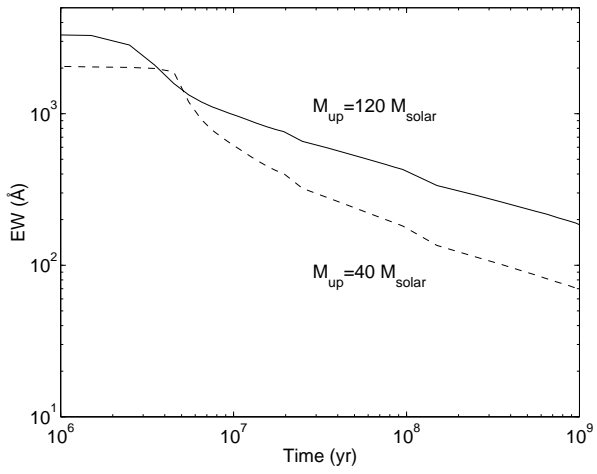

Figure 3 The equivalent width of $\mathrm{H} \alpha$ in emission from a starburst with constant SFR, a Salpeter IMF and $20 \%$ solar metallicity. The predicted evolution assuming two different values of the upper mass limit are shown. The model is from Zackrisson et al. (2001).

A very useful method to determine the past starburst activity in a galaxy is based on its rich system of super star clusters and globular clusters (GCs). The GC IMF is Salpeter-like and their stellar content is coeval. This makes them quite reliable as standard clocks and optical/nearIR photometry and spectroscopy can be used to determine their ages and trace past star formation history thereby identifying bursts (e.g. Östlin et al. 2003; de Grijs et al. 2004).

The best method to derive the ages is from colour-magnitude diagrams of the stellar population, but most starburst galaxies are to distant to make this method feasible. The few results available give no support for strong shortlived bursts separated by quiescent periods (Annibali et al. 2003, Schulte-Ladbeck et al. 2001). A similar conclusion was reached by Westera et al. (2004) in a study of 200 HII galaxies based on stellar absorption features. Taking the previous discussion into account, these observations indicate that true starbursts are rare rather than shortlived or that they are shortlived but change morphological type at or after the burst. 
The starburst host. It is well established that the luminosity profile of most BCGs can be characterised by a burst superposed on a host galaxy with (mostly) red colours, typical of an old stellar population, and a morphology resembling an early type galaxy (e.g. Papaderos et al. 1996, Gil de Paz et al. 2004). An attractive scenario is that a dE is merging with a gas rich galaxy that triggers the burst. Recently it was found that the optical/near-IR colours of the host of luminous BCGs at very faint levels has a red excess, difficult to explain with a normal IMF and a low metallicity (Bergvall \& Östlin 2002). This problem is discussed in the paper by Zackrisson et al. (2004). It could indicate that a host galaxy of special properties is needed to trigger a true starburst.

\section{References}

Annibali F., Greggio L., Tosi M., Aloisi A., Leitherer C., 2003, AJ, 126, 2752

Barton E. J., Geller M. J., Kenyon S. J., 2000, ApJ, 530, 660

Bergvall N., Östlin G., 2002, A\&A, 390, 891

Bergvall N., Laurikainen E., Aalto S., 2003, A\&A, 405, 31

Brosch N., Almoznino E., Heller A. B., 2004, MNRAS, 349, 357

de Grijs R., Smith L. J., Bunker A., et al., 2004, MNRAS, 352, 263

Elmegreen B. G., 2004, MNRAS, 354, 367

Gil de Paz A., Madore B. F., Pevunova O., 2003, ApJS, 147, 29

Hunter D. A., Elmegreen B. G., 2004, AJ, 128, 2170

Keel W. C., Kennicutt R. C., Hummel E., van der Hulst J. M., 1985, AJ, 90, 708

Knapen J. H., 2004, astro-ph/0407068

Lambas D. G., Tissera P. B., Alonso M. S., Coldwell G., 2003, MNRAS, 346, 1189

Larson R. B., Tinsley B. M., 1978, ApJ, 219, 46

Loose H.-H., Thuan F. X., 1986, MitAG, 65, 231

Marquart, T. et al., 2004, this volume

Östlin G., Amram P., Bergvall N., Masegosa J., Boulesteix J., Márquez I., 2001, A\&A, 374,800

Östlin G., et al., 2004, A\&A, 419, L43

Östlin G., Zackrisson E., Bergvall N., Rönnback J., 2003, A\&A, 408, 887

Papaderos P., Loose H.-H., Fricke K. J., Thuan T. X., 1996, A\&A, 314, 59

Salzer J. J., Rosenberg J. L., Weisstein E. W., Mazzarella J. M., Bothun G. D., 2002, AJ, 124, 191

Schaerer D., 2001, "Starburst Galaxies: Near and Far", Ringberg Castle, Ed.: L. Tacconi and D. Lutz., Springer, 197

Schulte-Ladbeck R. E., Hopp U., Greggio L., Crone M. M., Drozdovsky I. O., 2001, ApSSS, 277, 309

Varela J., Moles M., Márquez I., Galletta G., Masegosa J., Bettoni D., 2004, A\&A, 420,873

Westera P., Cuisinier F., Telles E., Kehrig C., 2004, A\&A, 423, 133

Zackrisson E., Bergvall N., Olofsson K., Siebert A., 2001, A\&A, 375, 814

Zackrisson, E., Bergvall, N., Marquart, T., Mattsson, L., Östlin, G., 2004, this volume 